\title{
A Topological Characterization of Classical BRST Cohomology
}

\author{
José M. Figueroa-O’Farrill*
}

Institute for Theoretical Physics, State University of New York at Stony Brook, Stony Brook, NY 11794-3840, USA

\begin{abstract}
The recent identification of classical BRST cohomology with the "vertical cohomology" of a certain fibration is used to compute it in terms of the classical observables and the topology of the gauge orbits. When the gauge orbits are compact and orientable, a duality theorem is exhibited.
\end{abstract}

\section{Introduction}

The classical BRST cohomology for finite dimensional systems has recently been interpreted geometrically. Let $(M, \Omega)$ be a symplectic manifold and $\left\{\phi_{i}\right\}$ a set of first class constraints. Their zero locus, $M_{0}=\cap_{i} \phi_{i}^{-1}(0)$, is a closed co-isotropic submanifold of $M$. The hamiltonian vector fields, $\left\{X_{i}\right\}$, associated to the constraints span the null directions of the restriction of $\Omega$ to $M_{0}$. Since this distributions is involutive, $M_{0}$ is foliated by maximal connected submanifolds having the $\left\{X_{i}\right\}$ as their tangent vectors. If this foliation, $\mathscr{F}$, fibers, the space of leaves $\tilde{M}$ can be given a differentiable structure such that the canonical map, $\pi: M_{0} \rightarrow \tilde{M}$, sending each point in $M_{0}$ to the unique leaf it lies in, is a smooth surjection. Moreover $\tilde{M}$ inherits a symplectic structure $\widetilde{\Omega}$, making $(\tilde{M}, \widetilde{\Omega})$ into a symplectic manifold. The passage from $(M, \Omega)$ to $(\tilde{M}, \widetilde{\Omega})$ is known as the symplectic reduction of $M$ by $M_{0}$.

The tangent bundle of $M_{0}$ breaks up as $T M_{0}=T \mathscr{F} \oplus N \mathscr{F}$, where $T \mathscr{F}=T M_{0}^{\perp}$ is the tangent space to the foliation and $N \mathscr{F}$ is the normal bundle to the foliation. Let $T^{*} \mathscr{F}$ and $N^{*} \mathscr{F}$ denote the cotangent and conormal bundles to the foliation, respectively. Under this split, the differential forms, $\Omega\left(M_{0}\right)$, on $M_{0}$ decompose as

$$
\Omega\left(M_{0}\right)=\underset{p, q}{\oplus} \Omega^{p, q}\left(M_{0}\right),
$$

where $\Omega^{p, q}\left(M_{0}\right)$ is the space of smooth sections through the bundle

$$
\wedge^{p} T^{*} \mathscr{F} \otimes \wedge^{q} N^{*} \mathscr{F} .
$$

\footnotetext{
^e-mail: figueroa@sunysbnp.BITNET,jmf@noether.UUCP
} 
The exterior derivative on $M_{0}$ has a piece

$$
d_{\mathscr{F}}: \Omega^{p, q} \rightarrow \Omega^{p+1, q},
$$

called the "vertical derivative," which corresponds to exterior differentiation along the leaves of the foliation. Let us define the "vertical forms" by $\Omega_{V}^{p}\left(M_{0}\right) \equiv \Omega^{p, 0}\left(M_{0}\right)$. They form a differential complex under the vertical derivative

$$
\ldots \rightarrow \Omega_{V}^{p}\left(M_{0}\right) \stackrel{d_{\mathscr{F}}}{\longrightarrow} \Omega_{V}^{p+1}\left(M_{0}\right) \rightarrow \ldots
$$

whose cohomology, called the "vertical cohomology," is precisely the cohomology of the classical BRST operator. This is proven in [1] and in [2] for the case of irreducible constraints; and in [3] for the case of reducible constraints.

In [4] the Poincare lemma for this complex is proven. That is, if $\omega$ is a $d_{\mathscr{F}}$-closed vertical $p$-form (for $p \geqq 1$ ), then around each point in $M_{0}$ there exists a neighborhood $U$ and a vertical $(p-1)$-form $\theta$ defined on $U$ such that $\omega=d_{\mathscr{F}} \theta$ on $U$. A vertical 0 -form is just a function on $M_{0}$ and it is $d_{\mathscr{F}}$-closed if and only if it is constant on each leaf. Therefore a $d_{\mathscr{F}}$-closed vertical 0 -form is the pull back via $\pi$ of a function on $\tilde{M}$. Let $\mathscr{E}_{\tilde{M}}$ be the sheaf of germs of smooth functions on $\tilde{M}$ and let $\Omega_{V}$ denote the sheaf of germs of vertical forms on $M_{0}$. By the above remarks there is an acyclic resolution

$$
0 \rightarrow \pi^{*} \mathscr{E}_{\tilde{M}} \rightarrow \Omega_{V}^{0} \stackrel{d_{\mathscr{F}}}{\longrightarrow} \Omega_{V}^{1} \rightarrow \ldots,
$$

where the first map is the inclusion. This identifies the vertical cohomology with the sheaf cohomology $H\left(M_{0} ; \pi^{*} \mathscr{E}_{\tilde{M}}\right)$ and thus makes contact with the work of Buchdahl [5] on the relative de Rham sequence, of which the vertical cohomology is an important special case.

Buchdahl treats the case of an arbitrary smooth surjective map $f: Y \rightarrow X$ between two arbitrary (smooth, paracompact) manifolds. He then obtains a resolution for the pull-back sheaf $f^{*} \mathscr{E}_{X}$ in terms of "relative" forms $\Omega_{f}$. Relative forms are differential forms along the "fibers" of $f$ and the derivative in the exterior derivative along the fibers. Hence vertical cohomology is a particular case of this construction for a very special $f, Y$, and $X$. Buchdahl does not characterize the relative cohomology completely, but he proves two results that relate it to the cohomology of the fibers. In the case of vertical cohomology, his results (Propositions 1 and 2 in [5]) imply the following two theorems, where $F$ is the typical fiber in the fibration $M_{0} \stackrel{\pi}{\longrightarrow} \tilde{M}$ and $H(F)$ stands for the real de Rham cohomology of the typical fiber.

Theorem 6. $H^{1}(F)=0$ implies $H_{V}^{1}\left(M_{0}\right)=0$. If $H^{p-1}(F)=H^{p}(F)=0$ for some $p>1$, then $H_{V}^{p}\left(M_{0}\right)=0$.

Theorem 7. If for some $p \geqq 1, H_{V}^{p}\left(M_{0}\right)=H_{V}^{p+1}\left(M_{0}\right)=0$, then $H^{p}(F)=0$.

An easy corollary of these two theorems gives a characterization of the vanishing of the BRST cohomology for positive ghost number.

Corollary 8. A necessary and sufficient condition for the classical BRST cohomology to vanish for positive ghost number is that the gauge orbits have vanishing positive de Rham cohomology. 
In particular in the case of a compact orientable gauge orbit, Poincare duality already forbids the vanishing of the BRST cohomology of top ghost number.

These results, although already providing a lot of information, are far from fully characterizing the BRST cohomology in terms of the topology of the gauge orbits and the gauge invariant observables. This is precisely the aim of this paper.

I will prove that for the case of a fibration

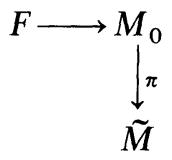

the vertical cohomology is isomorphic as $C^{\infty}(\tilde{M})$-modules to the space of smooth sections of a vector bundle over $\tilde{M}$ whose typical fiber is the real de Rham cohomology of $F$. This vector bundle is moreover an associated bundle to the original bundle via the natural representation

$$
\varrho: \operatorname{Diff} F \rightarrow \operatorname{Aut} H(F) \text {. }
$$

When the constraints arise from the hamiltonian group action of a connected Lie group $G$, the fibration $G \rightarrow M_{0} \rightarrow \tilde{M}$ is, in fact, a principal $G$-bundle and it follows that the associated bundle is trivial. In this case the BRST cohomology will simply be given by the $H(F)$-valued functions on $\tilde{M}$.

Finally, when the orbits are compact and orientable, the Poincaré duality on $H(F)$ induces a duality on the BRST cohomology. The orientability hypothesis is superfluous for irreducible constraints, since the fibers are parallelizable.

\section{Topological Characterization}

To fix the notation, let $F \rightarrow M_{0} \stackrel{\pi}{\rightarrow} \tilde{M}$ be a smooth fiber bundle where the typical fiber, $F$, is connected. Let $d_{V}$ denote the vertical derivative, $\Omega_{\mathrm{V}}\left(M_{0}\right)$ the vertical forms, and $H_{V}\left(M_{0}\right)$ the vertical cohomology. By definition, the zeroth vertical cohomology, $H_{V}^{0}\left(M_{0}\right)$, consists of those smooth functions on $M_{0}$ which are locally constant on the fibers; and since the fibers are connected, these functions are constant. The projection $\pi$ induces an isomorphism, $\pi^{*}: C^{\infty}(\tilde{M}) \rightarrow C^{\infty}\left(M_{0}\right)$, defined by $\pi^{*} f=f \circ \pi$, onto the smooth functions on $M_{0}$ which are constant on the fibers. Therefore, there is an isomorphism

$$
H_{V}^{0}\left(M_{0}\right) \cong C^{\infty}(\tilde{M}) .
$$

By its definition the vertical derivative $d_{V}$ obeys

$$
d_{V}(\omega \wedge \theta)=\left(d_{V} \omega\right) \wedge \theta+(-1)^{p} \omega \wedge\left(d_{V} \theta\right),
$$

for $\omega \in \Omega_{V}^{p}\left(M_{0}\right)$ and $\theta \in \Omega_{V}\left(M_{0}\right)$. Therefore $\wedge$ induces an operation in cohomology

$$
\cup: H_{V}^{p}\left(M_{0}\right) \times H_{V}^{q}\left(M_{0}\right) \rightarrow H_{V}^{p+q}\left(M_{0}\right),
$$

defined by $[\omega] \cup[\theta]=[\omega \wedge \theta]$. This operation is well defined because of $(12)$ and makes the vertical cohomology into a graded ring. In particular,

$$
\cup: H_{V}^{0}\left(M_{0}\right) \times H_{V}^{q}\left(M_{0}\right) \rightarrow H_{V}^{q}\left(M_{0}\right)
$$

makes $H_{V}\left(M_{0}\right)$ into a graded $H_{V}^{0}\left(M_{0}\right) \cong C^{\infty}(\tilde{M})$-module. 
Let $\mathscr{H}_{V}$ denote the sheaf of $C^{\infty}(\tilde{M})$-modules on $\tilde{M}$ defined by $\mathscr{H}_{V}(U)$ $=H_{V}\left(\pi^{-1} U\right)$ for all open $U \subset \tilde{M}$. By local triviality there exists an open cover $\mathscr{U}$ for $\tilde{M}$ such that for all $U \in \mathscr{U}, \pi^{-1} U \cong U \times F$. Therefore $\mathscr{H}_{V}(U) \cong H_{V}(U \times F)$. By a theorem of Kacimi-Alaoui (III(1) in [6]) the vertical cohomology of a product is given simply by

$$
H_{V}(U \times F) \cong C^{\infty}(U) \otimes H(F),
$$

where $H(F)$ is the real de Rham cohomology of $F$. This implies that $\mathscr{H}_{V}$ is a locally free sheaf and thus the sheaf of germs of smooth sections of a vector bundle over $\tilde{M}$ with fiber $H(F)$.

The task ahead is to determine the transition functions of this bundle. Let $\left\{\psi_{U}\right\}$ be the family of diffeomorphisms

$$
\psi_{U}: \pi^{-1} U \rightarrow U \times F
$$

given by the local triviality of the original bundle $M_{0} \stackrel{\pi}{\longrightarrow} \tilde{M}$. The transition functions of this bundle are then given, for all $U \cap V \neq \emptyset$, by $g_{U V}=\psi_{U} \circ \psi_{V}^{-1}$, thought of as a map $g_{U V}: U \cap V \rightarrow \operatorname{Diff} F$.

Recall that there is a natural representation of Diff $F$ as automorphisms of degree zero of the (graded) de Rham cohomology ring $H(F)$. If $\varphi \in \operatorname{Diff} F$ then the automorphism is defined by $[\omega] \mapsto\left[\left(\varphi^{-1}\right)^{*} \omega\right]$. By the homotopy invariance of de Rham cohomology, two diffeomorphisms which are homotopic are represented by the same automorphism in $H(F)$. So any diffeomorphism which is homotopic to the identity will automatically induce the identity automorphism on cohomology. maps

Composing the transition functions $\left\{g_{U V}\right\}$ with this representation provides

$$
\left(g_{U V}^{-1}\right)^{*}: U \cap V \rightarrow \operatorname{Aut} H(F),
$$

which, as I will now show, are the transition functions of the bundle whose sheaf of sections is given by $\mathscr{H}_{V}$.

To see this notice that for all open sets $U \in \mathscr{U}$,

$$
\left(\psi_{U}^{-1}\right)^{*}: H_{V}\left(\pi^{-1} U\right) \rightarrow H_{V}(U \times F) \cong C^{\infty}(\tilde{M}) \otimes H(F),
$$

allows us to identify vertical cohomology classes on $\pi^{-1} U$ with $H(F)$-valued functions on $U$. Let $\omega$ be a $d_{V}$-closed vertical form and $[\omega]$ its class in vertical cohomology. Restricted to $U \cap V$ there are two ways in which one can identify $[\omega]$ with an $H(F)$-valued function on $U \cap V$ : either by using the trivialization on $U$ or the one on $V$. Let $f_{U}=\left[\left(\psi_{U}^{-1}\right)^{*} \omega\right]$ and $f_{V}=\left[\left(\psi_{V}^{-1}\right)^{*} \omega\right]$. The transition functions $h_{U V}$ are precisely the automorphisms of the fiber $H(F)$ relating these two descriptions of the same object. That is, the transition functions obey $f_{U}=h_{U V} f_{V}$. But because

$$
\begin{aligned}
f_{U} & =\left[\left(\psi_{U}^{-1}\right)^{*} \omega\right]=\left[\left(\psi_{U}^{-1}\right)^{*} \circ \psi_{V}^{*} \circ\left(\psi_{V}^{-1}\right)^{*} \omega\right] \\
& =\left[\left(\psi_{U}^{-1}\right)^{*} \circ \psi_{V}^{*} f_{V}\right]=\left[\left(\psi_{V} \circ \psi_{U}^{-1}\right)^{*} f_{V}\right]=\left[\left(g_{U V}^{-1}\right)^{*} f_{V}\right],
\end{aligned}
$$

the transition functions are in fact the ones in (17). Therefore I have proven the following theorem. 
Theorem 20. The BRST cohomology is isomorphic as a $C^{\infty}(\tilde{M})$-module to the smooth sections of the associated bundle $M_{0} \times{ }_{e} H(F) \rightarrow \tilde{M}$ associated to the representation $\varrho: \operatorname{Diff} F \rightarrow \operatorname{Aut} H(F)$.

Notice that this associated bundle decomposes naturally as a Whitney sum of vector bundles

$$
M_{0} \times{ }_{Q} H(F)=\underset{p}{\oplus} M_{0} \times{ }_{e} H^{p}(F),
$$

since diffeomorphisms do not alter the degree of a form.

\section{The Case of a Group Action}

When the constraints arise from the hamiltonian action of a connected Lie Group $G$-i.e. the constraints are the coefficients of the moment map relative to a fixed basis for the Lie algebra of $G$ - the bundle

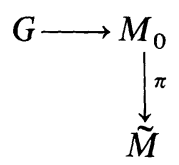

is in fact a principal $G$-bundle and the diffeomorphisms of $G$ defined by the transition functions correspond to right multiplication by an element of the group. Since $G$ is connected, right multiplication by any element $g \in G$ is homotopic to the identity. [Proof. Let $t \mapsto g(t)$ be a curve in $G$ such that $g(0)=1$ and $g(1)=g$. Right multiplication by $g(t)$ gives the desired homotopy.] By the homotopy invariance of de Rham cohomology, the transition functions of the associated bundle $M_{0} \times{ }_{e} H(G) \rightarrow \tilde{M}$ are the identity maps and thus the bundle is trivial. This proves the following corollary.

Corollary 23. When the constraints arise from the hamiltonian action of a connected Lie group $G$, the BRST cohomology is isomorphic to the $H(G)$-valued functions on $\tilde{M}$.

\section{The Case of Compact Orientable Fibers}

Finally suppose that the fibers are compact and orientable. ${ }^{1}$ Therefore Poincare duality induces an isomorphism

$$
*: H^{p}(F) \rightarrow H^{n-p}(F),
$$

where $n$ is the dimension of the fiber. This induces a duality in the BRST cohomology as follows. Let $\sigma$ be a section through $M_{0} \times{ }_{Q} H^{p}(F)$. Define a section $\tilde{*} \sigma$ through $M_{0} \times{ }_{Q} H^{n-p}(F)$ by

$$
(\approx \sigma)(m)=* \sigma(m) \quad \forall m \in \tilde{M} .
$$

This is an isomorphism and hence we have the following result.

\footnotetext{
${ }^{1}$ When the constraints are irreducible, the fibers are parallelizable (the $\left\{X_{i}\right\}$ are a global basis for the tangent space) and hence orientable
} 
Corollary 26. Let the typical fiber $F$ be $n$-dimensional, compact, and orientable. Then there is an isomorphism

$$
H_{V}^{p}\left(M_{0}\right) \cong H_{V}^{n-p}\left(M_{0}\right) .
$$

For some very interesting remarks concerning duality in BRST cohomology, see the recent paper [7].

Acknowledgements. It is a pleasure to thank Marc Henneaux for a most interesting correspondence on this and other related topics and for his comments on the manuscript. I would also like to thank Takashi Kimura for stimulating discussions, and Tony Phillips and Jim Stasheff for encouragement.

\section{References}

1. Dubois-Violette, M.: Ann. Inst. Fourier 37, 45 (1987)

2. Henneaux, M., Teitelboim, C.: Commun. Math. Phys. 115, 213 (1988)

3. Fisch, J., Henneaux, M., Stasheff, J., Teitelboim, C.: Existence, uniqueness, and cohomology of the classical BRST charge with ghosts of ghosts (Preprint, 1988)

4. Vaisman, I.: Czechosl. Math. 21, 46 (1971)

5. Buchdahl, N.: Am. Math. Soc. 87, 363 (1983)

6. El Kacimi-Alaoui, A.: Compositio Math. 49, 195 (1983)

7. Henneaux, M.: Duality theorems in BRST cohomology (Bruxelles Preprint, 1988)

Communicated by L. Alvarez-Gaumé

Received May 23, 1989 